\title{
Reversible Posterior Leukoencephalopathy Syndrome Due to Carboplatin and Paclitaxel Therapy
}

\author{
Melek Kandemir ${ }^{1}$, Belgin Küçükkaya ${ }^{2}$, Muzaffer Savaş Tepe ${ }^{3}$, Zehra Betül Yalçıner ${ }^{1}$, Nedret Taflan Salepçi ${ }^{4}$
}

\author{
${ }^{1}$ Department of Neurology, Bayındır Hospital İçerenköy, İstanbul, Turkey \\ ${ }^{2}$ Department of Internal Medicine, Bayındır Hospital İçerenköy, İstanbul, Turkey \\ ${ }^{3}$ Department of Radiology, Bayındır Hospital İçerenköy, İstanbul, Turkey \\ ${ }^{4}$ Department of Oncology, Italian Hospital, İstanbul, Turkey
}

Background: Reversible posterior leukoencephalopathy syndrome (RPLS) is a clinicoradiologic syndrome characterized by headache, decreased alertness, seizures, visual abnormalities, and white matter changes indicative of cerebral edema. Although the pathogenesis remains poorly understood, several etiological causes have been described. RPLS is a common complication of chemotherapeutics because of its toxic effect on the central nervous system. This syndrome is frequently associated with seizures but rarely seen with status epilepticus and periodic lateralized epileptiform discharges (PLEDs).
Case Report: We present a case with metastatic lung cancer that developed RPLS after carboplatin and paclitaxel therapy. Our case was admitted to the hospital with status epilepticus and her electroencephalography showed PLEDs.

Conclusion: It is important to closely monitor blood pressure and electrolyte levels in patients who take chemotherapeutic agents, especially when there is no previous history of hypertension. It should be kept in mind that RPLS is a causative factor of status epilepticus and PLEDs.

Keywords: Carboplatin, drug therapy, paclitaxel, posterior leukoencephalopathy syndrome, status epilepticus
Reversible posterior leukoencephalopathy syndrome (RPLS) was first described by Hinchey et al. (1) in 1996. This is a clinicoradiologic syndrome characterized by headache, decreased alertness, altered behavior ranging from drowsiness to stupor, seizures, abnormalities of visual perception, and white matter changes indicative of cerebral edema on magnetic resonance imaging (MRI) and computerized tomography (CT), especially in bilateral posterior parietal-temporal-occipital regions of the brain (1). CT and MRI showed extensive bilateral white-matter abnormalities suggestive of edema in the posterior regions of the cerebral hemispheres, but the changes may involve other cerebral areas, the brainstem, or the cerebellum (1). Although the pathogenesis remains poorly understood, hypertensive encephalopathy, preeclampsia or ec- lampsia, renal disease, infection, several immunosuppressant agents, chemotherapeutic agents as mono- or combined therapy are known etiological causes of RPLS (1-8). Recently, a case has been reported with RPLS associated with carboplatin and paclitaxel regimen in a lung cancer patient from Japan (8). We present a case with metastatic lung cancer that developed RPLS after carboplatin and paclitaxel therapy.

\section{CASE PRESENTATION}

A 60 year-old woman with a past smoking history was being followed due to lung cancer stated in the right upper lobe, stage IV, with metastases in the liver, bone marrow, and bilat- 
eral adrenal glands. She had paraneoplastic Cushing syndrome and her electrolyte imbalance was corrected. No cranial metastases were detected on CT. She was treated with cisplatin $(40 \mathrm{mg})$ and etoposide $(180 \mathrm{mg})$ for 6 cycles. After 6 cycles, she took prophylactic radiotherapy to the cranium. She was treated with cisplatin $(50 \mathrm{mg})$ and irinocetan $(140 \mathrm{mg})$ for 2 cycles. This treatment was stopped because of diarrhea due to irinocetan and radiotherapy to the pelvis. After that, paclitaxel $\left(175 \mathrm{mg} / \mathrm{m}^{2}\right)$ and carboplatin (6 AUC $\min \cdot \mathrm{mg} / \mathrm{mL}$ ) treatment was initiated. Eight $\mathrm{mg}$ of intravenous dexamethasone was administered as an antiemetic medication during chemotherapy cycles. Six days after the $3^{\text {rd }}$ cycle of the carboplatin and paclitaxel regimen, she was admitted to the emergency department because of generalized tonic-clonic seizures - twice in twelve hours - and loss of consciousness. Her pupils were mid-dilated, direct and indirect light reflexes were positive. No gaze paralysis or facial asymmetry was seen. She had localized painful stimuli. The Babinski sign was detected bilaterally. Her vital signs on admission were: blood pressure $190 / 100 \mathrm{mmHg}$, temperature $36.7^{\circ} \mathrm{C}$, and cardiac pulse $112 / \mathrm{min}$. Blood tests were normal except for the increased lactate dehydrogenase and gamma glutamyl transferase and decreased magnesium levels $(\mathrm{Mg}=0.83 \mathrm{mg} / \mathrm{dL})$. She had repeated seizures at the emergency department and during CT scanning. The patient was treated with IV phenytoin infusion for the status epilepticus. MRI with gadolinium revealed neither evidence of brain metastasis nor pathologic enhancement. However, MRI showed bilateral abnormal white matter hyperintensities in the parietal and occipital lobes in T2 weighted and fluid attenuation inversion recovery (FLAIR) sequences suggestive of edema in the regions of gray matter-white matter junction (Figure 1). T1 weighted imaging showed hypointensities in the corresponding areas. Diffusion weighted imaging (DWI) showed increased signal intensity and apparent diffusion coefficient (ADC) mapping showed decreased signal intensity in the corresponding subcortical regions (Figure 2a and b. According to clinical and radiological findings, the patient was diagnosed with RPLS. On the day following admission to the hospital, electroencephalography (EEG) showed slowing of the posterior background composed of delta and theta waves. On the $4^{\text {th }}$ day of admission, EEG showed slowing of the posterior background and periodic lateralized epileptiform discharges (PLEDs) on both temporo-parieto-occipital regions more prominent over the left hemisphere (Figure 3). While her condition gradually improved with supportive therapy, corticosteroids and antiepileptic treatment, she became non-cooperative and agitated. The patient was only able to say unpurposeful words and only responded to simple commands. She had cortical blindness with response only to light. Her limbs were able to move spontaneously and symmetrically. There

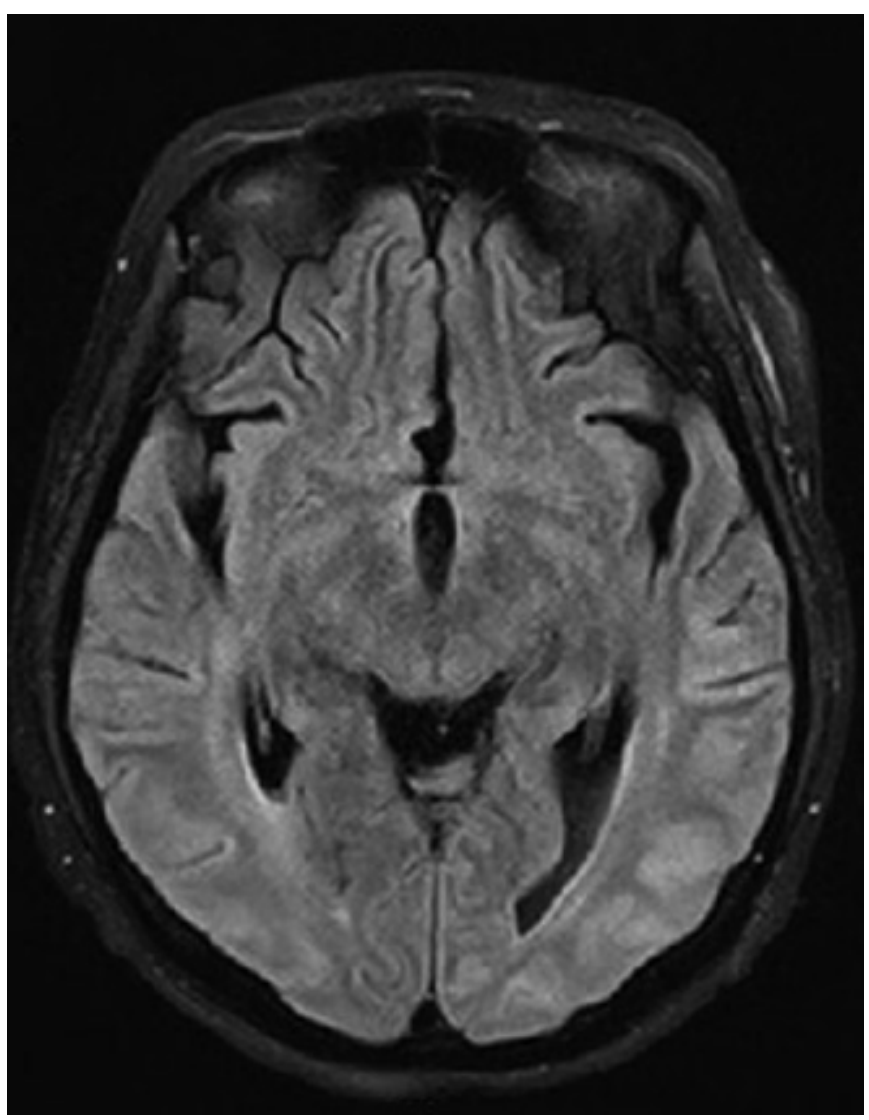

FIG. 1. Fluid attenuated inversion recovery MRI sequence shows bilateral, predominantly posterior, gyral subcortical and cortical hyperintense severe signal abnormalities on both parietal occipital lobes more on the left side representing extensive vasogenic edema.

was no Babinski sign. She had visual hallucinations. She was treated with $16 \mathrm{mg} / \mathrm{g}$ dexamethasone for RPLS. Dexamethasone was tapered according to her clinical status, EEG and radiological findings. One week later, she became cooperative and her vision improved. Her visual hallucinations continued for about 10 days. No seizures were observed after phenytoin infusion which was given on the first day. The patient was discharged from hospital on the $10^{\text {th }}$ day. She was treated with $750 \mathrm{mg} / \mathrm{d}$ levetiracetam for seizures. Her neurological examination findings returned to normal except for the agitation and decreased vision. Follow-up MRI scanning performed in the $3^{\text {rd }}$ and $6^{\text {th }}$ weeks showed gradual resolution of the described hyperintense lesions. On the $17^{\text {th }}$ and $50^{\text {th }}$ days of the syndrome, EEGs showed slowing of the background and PLEDs on both temporo-parieto-occipital regions. She underwent a $4^{\text {th }}$ cycle of carboplatin and paclitaxel therapy after 17 days. Thirty-one days after discharge, the patient died secondary to cardiopulmonary arrest due to cancer progression. 

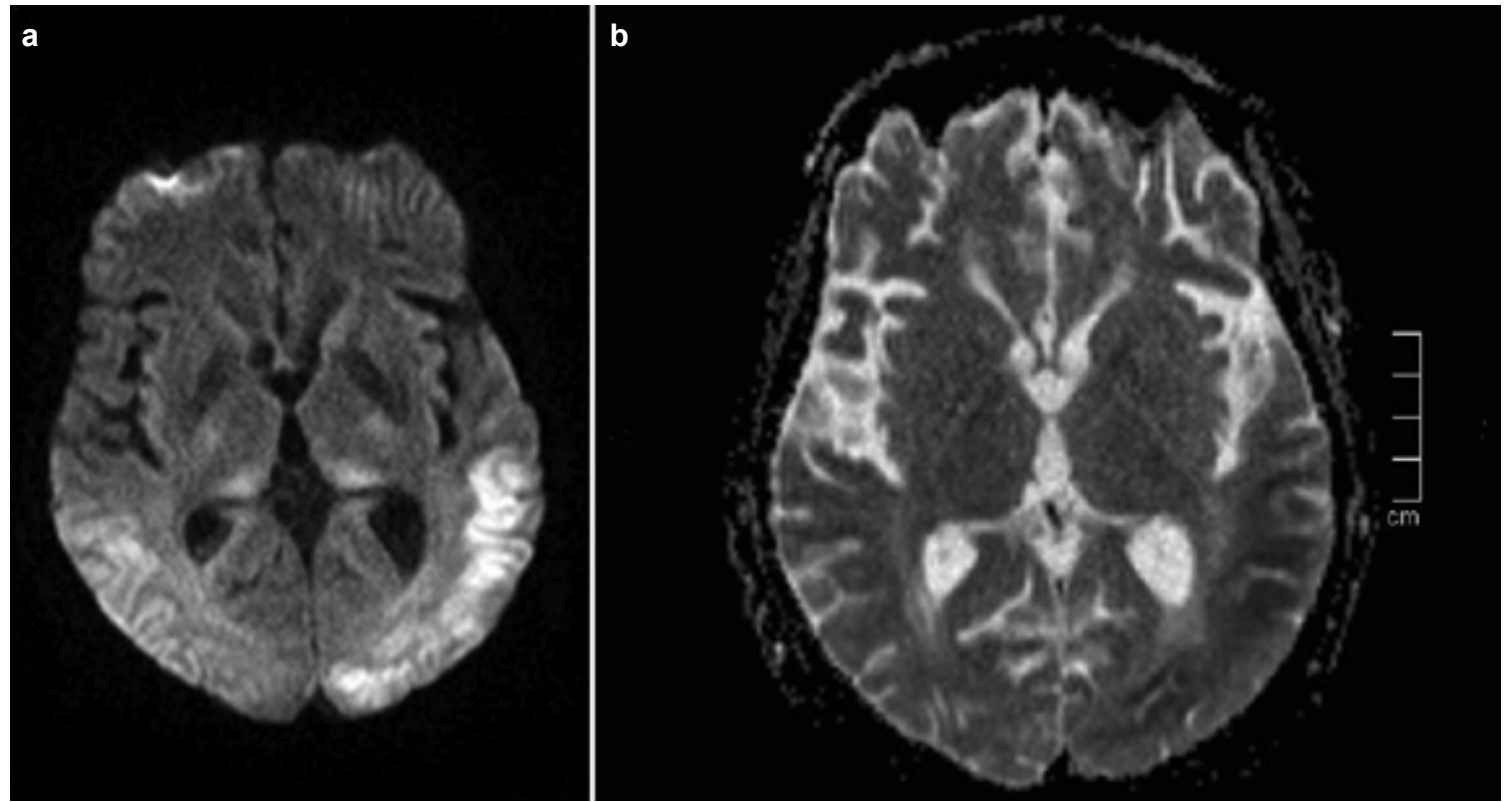

FIG. 2. a, b. DWI sequence reveals restricted diffusion abnormalities at the corresponding area mainly involving the left cerebral hemisphere (a). $A D C$ sequence reveals decreased signal intensity in the corresponding area mainly involving the left cerebral hemisphere (b).

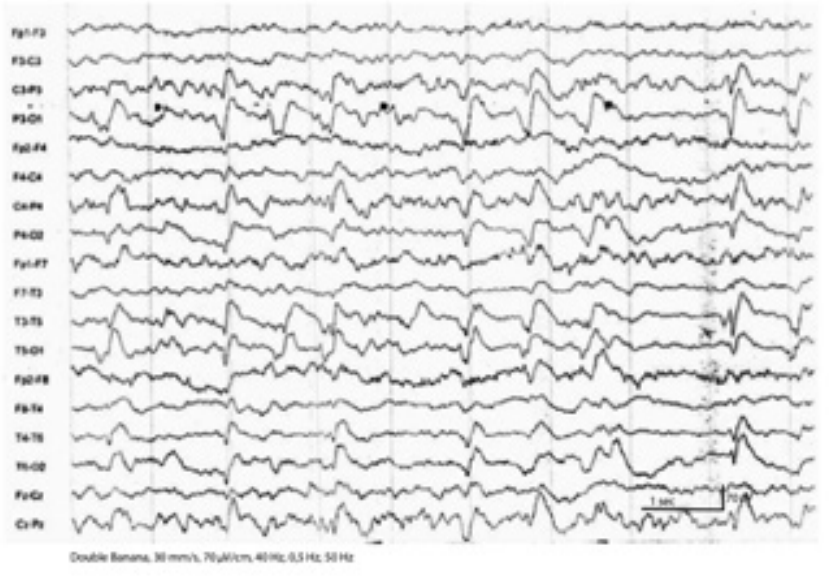

FIG. 3. EEG demonstrating the slowing of the posterior background and posteriorly dominant bilateral PLEDs. PLEDs were higher in amplitude over the left hemisphere. (LPF: $40 \mathrm{~Hz}, \mathrm{HPF}: 0.5 \mathrm{~Hz}, 70 \mu \mathrm{V} /$ $\mathrm{cm}, 30 \mathrm{~mm} / \mathrm{s}$ )

\section{DISCUSSION}

The most common clinical presentations of RPLS are newonset seizures, encephalopathy, headache, and visual disturbances (7). Seizures can be seen at the onset of the neurological symptoms and can begin focally, but usually become generalized (1). Status epilepticus is an uncommon clinical presentation of RPLS (7). In this case, we considered RPLS to be related to the carboplatin and paclitaxel regimen according to the patient's previous clinical status and radiological find- ings. The patient's seizures began 6 days after the $3^{\text {rd }}$ cycle of chemotherapy. The symptoms of RPLS due to chemotherapeutic agents usually begin 1 or 2 weeks after the chemotherapy cycle $(2,3,5,9)$. RPLS is frequently associated with seizures but rarely seen with status epilepticus and PLEDs $(6,7,10,11)$. Kozak et al. (10) reported a series of 10 patients with RPLS and status epilepticus. The majority of them showed resolution with standard antiepileptic treatment. Our patient was admitted to the hospital with status epilepticus and her seizures ceased after phenytoin infusion. PLEDs were determined on both parieto-occipital regions and these were correlated with the imaging findings. Skiba et al. (11) state that RPLS associated with PLEDs may cause recurrent seizures. Although PLEDs were seen on the $4^{\text {th }}, 17^{\text {th }}$ and $50^{\text {th }}$ days of RPLS, no deterioration was observed on clinical and radiological findings.

Immunosuppressive and chemotherapeutic drugs can cause neurological abnormalities, such as encephalopathy syndromes, confused states, seizures, headache, cerebrovascular complications, visual loss, cerebellar dysfunction, and spinal cord damage with myelopathy. RPLS is a common complication of chemotherapeutics because of its toxic effect to the central nervous system (12). The mechanism of RPLS is thought to be cerebral vasogenic edema related to hypertension and brain-capillary leak syndrome, failure of vascular auto-regulation, and possibly the cytotoxic effects of immunosuppressive and chemotherapeutic agents on the vascular endothelium and damage to the blood-brain barrier $(1,3,5)$. Platinum analogues are common chemotherapeutic agents used for solid or hematologic malignancies and central nervous system toxic- 
ity has been reported (13). It has occasionally been reported with cisplatin and rarely with carboplatin and oxaliplatin $(9,13)$. Imai et al. (8) recently reported a case with RPLS after carboplatin and paclitaxel regimen and stated that they considered this regimen to be associated with toxic damage to the vascular endothelium. The time to onset of RPLS by those agents may occur within several hours to months. The toxicity usually occurs shortly after the cessation of cisplatin administration, and often when the total dose exceeds $200 \mathrm{mg} / \mathrm{m}^{2}$ (14). This toxicity may not preclude further treatment with cisplatin $(9,14)$.

The characteristic imaging pattern in RPLS is the presence of an edema involving the white matter of the posterior portions of both cerebral hemispheres, especially the parieto-occipital regions, in a relatively symmetric pattern that spares the calcarine and paramedian parts of the occipital lobes (1). Tam et al. (4) pointed out that patients with RPLS often present with only moderate levels of hypertension in contrast to encephalopathy caused by isolated malignant hypertension. When the blood pressure rises modestly, hyperperfusion of the brain due to dysfunction of the cerebral vascular auto-regulation in RPLS can result in interstitial edema $(1,4)$. Onujiogu et al. (15) reported a case with RPLS in conjunction with hypertension after one cycle of intravenous paclitaxel followed by intraperitoneal cisplatin. They emphasized that hypertension should be controlled in patients prior to receiving this chemotherapy regimen due to potential toxicity (15). However, Tam et al. (4) suggested that RPLS has no or little relation to hypertension. Consistent with this, in the series by Fugate et al. (7), $46 \%$ of patients had no previous hypertension. In our patient, blood pressure levels were under control with medical treatment. Her blood pressure was high when the neurological symptoms began. An acute rise in blood pressure is more important than the overall blood pressure level and most of the patients' blood pressures were high at the time of admission (16). We think that, in some patients including ours, hypertension may not be the causative or predicting factor; instead, it had simply increased as a result of the ongoing pathogenesis.

Tam et al. (4) affirmed that patients with significant fluid overload (above 10\% baseline weight), mean blood pressure above $25 \%$ of baseline, and creatinine level of more than 0.16 $\mathrm{mmol} / \mathrm{L}$ may be at high risk of RPLS. Our patient's serum creatinine level was normal and she was not hypervolemic. When she was admitted to the hospital, her laboratory findings showed normocalcemia and hypomagnesemia. She had taken replacement therapy for hypokalemia and hypocalcemia. Hypomagnesemia was detected in series such as Ito et al. and Gorman et al. $(2,14)$; these authors thought that hypomagnesemia was a contributing factor for RPLS in cisplatin-related encephalopathy $(2,14)$. Correction of possible fluid and elec- trolyte imbalances is important in these cases, as the replacement of electrolytes, especially magnesium, may prevent the severe course of the syndrome. Ito et al. (2) suggested that magnesium could be the drug of choice in the treatment.

It is important to closely monitor patients taking chemotherapeutic agents with regard to blood pressure and electrolyte levels, especially in those with no previous history of hypertension. Correction of any electrolyte imbalances should be made immediately. It should be kept in mind that RPLS is a causative factor of status epilepticus and PLEDs.

\section{Ethics Committee Approval: N/A.}

Informed Consent: Written informed consent was obtained from the patient's relative who participated in this case.

Peer-review: Externally peer-reviewed.

Author contributions: Concept - M.K., B.K.; Design - M.K., B.K.; Supervision - Z.B.Y., N.T.S.; Resource -M.K.; Data Collection \&/or Processing - M.K., B.K., M.S.T., N.T.S., Z.B.Y.; Analysis \&/ or Interpretation - M.K.; Literature Search - M.K.; Writing - M.K., B.K., M.S.T.; Critical Reviews - M.K., B.K., M.S.T., Z.B.Y., N.T.S.

Conflict of Interest: No conflict of interest was declared by the authors.

Financial Disclosure: The authors declared that this study has received no financial support.

\section{REFERENCES}

1. Hinchey J, Chaves C, Appignani B, Breen J, Pao L, Wang A, et al. A reversible posterior leukoencephalopathy syndrome. $N$ Engl J Med 1996;334:494-500. [CrossRef]

2. Ito Y, Arahata Y, Goto Y, Hirayama M, Nagamutsu M, Yasuda $\mathrm{T}$, et al. Cisplatin neurotoxicity presenting as reversible posterior leukoencephalopathy syndrome. AJNR Am J Neuroradiol 1998;19:415-7.

3. Edwards MJ, Walker R, Vinnicombe S, Barlow C, MacCallum P, Foran JM. Reversible posterior leukoencephalopathy syndrome following CHOP chemotherapy for diffuse large B-cell lymphoma. Ann Oncol 2001;12:1327-9. [CrossRef]

4. Tam CS, Galanos J, Seymour JF, Pitman AG, Stark RJ, Prince HM. Reversible posterior leukoencephalopathy syndrome complicating cytotoxic chemotherapy for hematologic malignancies. Am J Hematol 2004;77:72-6. [CrossRef]

5. Rajasekhar A, George TJ Jr. Gemcitabine-induced reversible posterior leukoencephalopathy syndrome: A case report and review of literature. Oncologist 2007;12:1332-5. [CrossRef]

6. Bhatt A, Farooq MU, Bhatt S, Majid A, Kassab MY. Periodic lateralized epielptiform discharges: an initial electrographic 
pattern in reversible posterior leukoencephalopathy syndrome. Neurol Neurochir Pol 2008;42:55-9.

7. Fugate JE, Claassen DO, Cloft HJ, Kallmes DF, Kozak OS, Rabinstein AA. Posterior reversible encephalopathy syndrome: associated clinical and radiological findings. Mayo Clin Proc 2010;85:427-32. [CrossRef]

8. Imai H, Okuno N, Ishihara S, Nakano S, Higuchi S, Arai T, et al. Reversible posterior leukoencephalopathy syndrome after carboplatin and paclitaxel regimen for lung cancer. Intern Med 2012;51:911-5. [CrossRef]

9. Vieillot S, Pouessel D, de Champfleur NM, Becht C, Culine S. Reversible posterior leukoencephalopathy syndrome after carboplatin therapy. Ann Oncol 2007;18:608-9. [CrossRef]

10. Kozak OS, Wijdicks EF, Manno EM, Miley JT, Rabinstein AA. Status epilepticus as initial manifestation of posterior reversible encephalopathy syndrome. Neurology 2007;69:894-7. [CrossRef]

11. Skiba V, Etienne M, Miller JA. Development of chronic epilepsy after recurrent episodes of posterior reversible leukoencepha- lopathy syndrome associated with periodic lateralized epileptiform discharges. Seizure 2011;20:93-5. [CrossRef]

12. Newton HB. Neurological complications of chemotherapy to the central nervous system. Handb Clin Neurol 2012;105:90316. [CrossRef]

13. Sharief U, Perry DJ. Delayed reversible posterior encephalopathy syndrome following chemotherapy with oxaliplatin. Clin Colorectal Cancer 2009;8:163-5. [CrossRef]

14. Gorman DJ, Kefford R, Stuart-Harris R. Focal encephalopathy after cisplatin therapy. Med J Aust 1989;150:399-401.

15. Onujiogu N, Lengyel E, Yamada SD. Reversible posterior leukoencephalopathy syndrome following intravenous paclitaxel and intraperitoneal cisplatin chemotherapy for fallopian tube cancer. Gynecol Oncol 2008;111:537-9. [CrossRef]

16. Bartynski WS. Posterior reversible encephalopathy syndrome, part 1: fundamental imaging and clinical features. AJNR Am J Neuroradiol 2008;29:1036-42.[CrossRef] 